\title{
SOME REMARKS ABOUT SYMMETRIC FUNCTIONS
}

\author{
EDGAR H. BROWN, JR. AND FRANKLIN P. PETERSON ${ }^{1}$
}

Abstract. A formula is proven which determines whether or not a symmetric function is decomposable. Some applications to topology are mentioned.

1. Statement of results. Let $Z\left[t_{1}, \ldots, t_{n}\right]$ be the polynomial ring in $n$ variables over the integers. Let $\omega=\left(i_{1}, \ldots, i_{k}\right)$ be a partition of $n$. Let $s_{\omega}$ be the smallest symmetric function containing the term $t_{1}^{i_{1}} \ldots t_{k}^{i_{k}}$. It is classical that $s_{\omega}$ is a polynomial in the elementary symmetric functions $\sigma_{1}, \ldots, \sigma_{n}$; i.e. $s_{\omega}=S_{\omega}\left(\sigma_{1}, \ldots, \sigma_{n}\right)$. Corresponding to $\omega$, we define a sequence $R(\omega)=\left(r_{1}\right.$, $\left.r_{2}, \ldots\right)$, by $r_{j}=$ the number of $j$ 's in $\omega$. Let $r=\Sigma_{j} r_{j}$. Note that $n=\Sigma_{j} r_{j}$. For various applications in topology, we wish to know whether or not $S_{\omega}$ is decomposable, i.e. whether or not the coefficient of $\sigma_{n}$ in $S_{\omega}$ is zero (or $\equiv 0 \bmod p$ ). Our first theorem solves this problem.

THEOREM 1.1.

$$
\frac{\partial S_{\omega}}{\partial \sigma_{n}}=\frac{(-1)^{n-r}(r-1) ! n}{r_{1} ! r_{2} ! \ldots} .
$$

After we proved this result, it was pointed out to us that it is stated without proof by Atiyah and Todd [1]. Our proof is elementary and we believe that the method may be of interest in its own right.

Let $A$ be the matrix whose entry

$$
A_{i j}=\frac{\partial \sigma_{i}}{\partial t_{j}}=\sum_{k_{s} \neq j} t_{k_{1}} t_{k_{2}} \ldots t_{k_{i-1}}
$$

Let $C(i \mid j)$ be the matrix obtained from $A$ be removing the $i$ th row and the $j$ th column.

THEOREM 1.2.

$$
\operatorname{det} A=\sum_{\tau \in \delta_{n}}(\operatorname{sgn} \tau) t_{\tau(1)}^{n-1} t_{\tau(2)}^{n-2} \ldots t_{\tau(n-1)}=\prod_{i<j}\left(t_{i}-t_{j}\right)
$$

THEOREM 1.3.

$$
\operatorname{det} C(n \mid k)=\prod_{\substack{i<j \\ i, j \neq k}}\left(t_{i}-t_{j}\right)=\sum_{\tau \in \delta_{n-1}}(\operatorname{sgn} \tau) t_{\tau(1)}^{n-2} t_{\tau(2)}^{n-3} \ldots t_{\tau(n-2)},
$$

$\delta_{n-1}$ being permutations of $t_{1}, \ldots, \hat{t}_{k}, \ldots, t_{n}$.

Received by the editors November 27, 1975.

AMS (MOS) subject classifications (1970). Primary 55F45.

Key words and phrases. Symmetric function, determinants, chain rule.

${ }^{1}$ The authors were partially supported by the National Science Foundation. 
THEOREM 1.4 .

$$
\sum_{k=1}^{n}(-1)^{n+k} \frac{\partial s_{\omega}}{\partial t_{k}} \operatorname{det} C(n \mid k)=\frac{\partial S_{\omega}}{\partial \sigma_{n}} \operatorname{det} A .
$$

In $\$ 2$ we prove Theorems $1.2,1.3$, and 1.4 and then use these theorems in $\S 3$ to prove Theorem 1.1. In $\S 4$, we mention a few topological applications.

2. Determinants. The following lemma is an elementary fact about polynomials.

Lemma 2.1. Let $P \in Z\left[t_{1}, \ldots, t_{n}\right]$ be a homogeneous polynomial of degree $(n-1) n / 2$. Assume $P\left(t_{1}, \ldots, t_{i}, \ldots, t_{j-1}, t_{i}, t_{j+1}, \ldots, t_{n}\right)=0$ for all $i, j$. Then $P$ is a scalar multiple of $\Pi_{i<j}\left(t_{i}-t_{j}\right)$.

Theorem 1.2 follows from this lemma if we show that both $\operatorname{det} A$ and $\Sigma_{\tau \in \delta_{n}}(\operatorname{sgn} \tau) t_{\tau(1)}^{n-1} t_{\tau(2)}^{n-2} \ldots t_{\tau(n-1)}$ satisfy the hypotheses of 2.1 and that both contain the term $t_{1}^{n-1} \ldots t_{n-1}$.

Lemma 2.2. $\operatorname{det} A$ satisfies the hypotheses of 2.1 and contains the term $t_{1}^{n-1} \ldots t_{n-1}$.

Proof. If $t_{j}=t_{i}$, then two columns of $A$ are the same and hence $\operatorname{det} A=0$. The degree is correct and the product down the diagonal is the only one to contain $t_{1}^{n-1} \ldots t_{n-1}$ and it has coefficient +1 .

LEMMA 2.3. $\sum_{\tau \in \delta_{n}}(\operatorname{sgn} \tau) t_{\tau(1)}^{n-1} \ldots t_{\tau(n-1)}$ satisfies the hypotheses of 2.1 and contains the term $t_{1}^{n-1} \ldots t_{n-1}$.

Proof. This polynomial has the correct degree and contains the term stated when $\tau$ is the identity. Let $\tau^{\prime} \in \mathcal{S}_{n}$ be the permutation which interchanges $i$ and $j . \operatorname{sgn} \tau^{\prime}=-1$; hence the terms for $\tau$ and $\tau^{\prime} \tau$ are the same but with opposite signs and thus they cancel. This proves the lemma.

Theorem 1.3 is proved in an analogous way to Theorem 1.2 with modified forms of Lemmas 2.1, 2.2, and 2.3. We leave the details to the reader.

We now prove Theorem 1.4. Recall that the classical adjoint of the matrix $A$ is defined by

$$
(\operatorname{adj} A)_{i j}=(-1)^{i+j} \operatorname{det} C(j \mid i),
$$

and that $A(\operatorname{adj} A)=(\operatorname{adj} A) A=(\operatorname{det} A) I$.

$$
\frac{\partial s_{\omega}}{\partial t_{k}}=\sum_{i} \frac{\partial S_{\omega}}{\partial \sigma_{i}} \cdot \frac{\partial \sigma_{i}}{\partial t_{k}}
$$

by the chain rule. Multiply by $(\operatorname{adj} A)_{k n}$ and sum on $k$.

$$
\sum_{k} \frac{\partial s_{\omega}}{\partial t_{k}}(-1)^{n+k} \operatorname{det} C(n \mid k)=\sum_{i, k} \frac{\partial S_{\omega}}{\partial \sigma_{i}} A_{i k}(\operatorname{adj} A)_{k n}=\frac{\partial S_{\omega}}{\partial \sigma_{n}} \operatorname{det} A .
$$

3. Proof of 1.1. In order to prove 1.1, we consider the left-hand side of Theorem 1.4 and count how many times the term $t_{1}^{n-1} \ldots t_{n-1}$ appears. Since it appears only once in $\operatorname{det} A$, this number must be $\partial S_{\omega} / \partial \sigma_{n}$. Consider the term $\left(\partial s_{\omega} / \partial t_{k}\right) t_{i_{1}}^{n-2} t_{i_{2}}^{n-3} \ldots t_{i_{n-2}}$, where $k \neq i_{j}$. For each $s$, the term $t_{j_{1}}^{s} t_{j_{2}}^{s} \ldots t_{j_{r_{s}}}^{s}$ in $s_{\omega}$ must have $j_{1}<\cdots \stackrel{<}{<} j_{r_{s}}$. Hence only $k=j_{r_{s}}$ can give rise to terms 
$t_{1}^{n-1} \ldots t_{n-1} \cdot t_{j_{s}}^{s-1}$ appears, hence $j_{r_{s}}=n-s+1, i_{n-2}=n-1, i_{n-3}=n-$ $2, \ldots, j_{r_{s}}$ is the only term which is fixed. The other $(r-1)$ variables are shuffled, $r_{1}, r_{2}, \ldots, r_{s-1},\left(r_{s}-1\right), r_{s+1}, \ldots$ at a time. Hence for each $s$, the number of times the term $t_{1}^{n-1} \ldots t_{n-1}$ appears is

$$
\frac{(r-1) ! s}{r_{1} ! \ldots\left(r_{s-1}\right) !\left(r_{s}-1\right) !\left(r_{s+1}\right) ! \ldots}
$$

with signs yet to be determined. The sign of $\tau \in \mathcal{S}_{n-1}$ corresponding to the shuffle is $(-1)^{n-s-(r-1)}$. There is also $(-1)^{n+k}=(-1)^{n+n-s+1}$ from the left-hand side of 1.4. Hence, the total sign is $(-1)^{3 n-2 s-r+2}=(-1)^{n-r}$. Thus, the total number of terms is

$$
\begin{aligned}
(-1)^{n-r} \sum_{s} \frac{(r-1) ! s}{r_{1} ! \ldots\left(r_{s}-1\right) ! \ldots} & =(-1)^{n-r} \sum_{s} \frac{(r-1) ! s r_{s}}{r_{1} ! \ldots} \\
& =(-1)^{n-r} \frac{(r-1) ! n}{r_{1} ! \ldots} .
\end{aligned}
$$

This proves 1.1 .

4. Some applications. Our original interest in this problem (before we were aware of the result in [1]) came from the following immediate corollary of 1.1. We needed this result in our first proof of the main results of [3], and it will be used in [2].

COROLlaRY 4.1. In $Z\left[t_{1}, \ldots, t_{n}\right], \partial S_{(i, i, \ldots, i)} / \partial \sigma_{m i}=(-1)^{(i-1) m_{i}}$.

Proof. If there are $m i$ 's, then

$$
(-1)^{i m-m}(m-1) ! i m / m !=(-1)^{(i-1) m} i .
$$

This is useful in topology because $H^{*}\left(B O(n) ; Z_{2}\right)$ is isomorphic to the symmetric functions in $Z_{2}\left[t_{1}, \ldots, t_{n}\right]$. Similarly, $H^{*}\left(B U(n) ; Z_{p}\right)$ is isomorphic to the symmetric functions in $Z_{p}\left[t_{1}, \ldots, t_{n}\right]$. We also have the following corollary (see [4]).

COROllaRY 4.2. Let $c_{n} \in H^{2 n}\left(B U ; Z_{p}\right)$ be the nth Chern class. Then

$$
\rho^{k}\left(c_{n}\right)=\left(\begin{array}{c}
n-1 \\
k
\end{array}\right) c_{n+k(p-1)}+\text { decomposables. }
$$

Proof. $c_{n}=\sigma_{n} \in Z_{p}\left[t_{1}, \ldots, t_{n+k(p+1)}\right], \rho^{k}\left(c_{n}\right)=\sum t_{1}^{p} \ldots t_{k}^{p} t_{k+1} \ldots t_{n}$. Hence,

$$
\begin{aligned}
\frac{\partial \mathscr{P}^{k}\left(c_{n}\right)}{\partial \sigma_{n+k(p-1)}} & =(-1)^{n+k(p-1)-n} \frac{(n+k(p-1)) \cdot(n-1) !}{k !(n-k) !} \\
& =\left(\begin{array}{l}
n \\
k
\end{array}\right)+(p-1)\left(\begin{array}{l}
n-1 \\
k-1
\end{array}\right)=\left(\begin{array}{c}
n-1 \\
k
\end{array}\right)+p\left(\begin{array}{l}
n-1 \\
k-1
\end{array}\right) \equiv\left(\begin{array}{c}
n-1 \\
k
\end{array}\right) .
\end{aligned}
$$

\section{REFERENCES}

1. M. F. Atiyah and J. A. Todd, On complex Stiefel manifolds, Proc. Cambridge Philos. Soc. 56 (1960), 342-353. MR 24 \# A2392.

2. E. H. Brown, Jr., D. Davis and F. P. Peterson (to appear). 
3. E. H. Brown, Jr. and F. P. Peterson, $H^{*}(M O)$ as an algebra over the Steenrod algebra (Reunion Sobre Teoria de Homotopia, Univ. de Northwestern, 1974), Bol. Soc. Mat. Mexicana (to appear).

4. S. Mukohda and S. Sawaki, On the $b_{p}^{k j}$ coefficient of a certain symmetric function, J. Fac. Sci. Niigata Univ. Ser. I, 1 (1954), no. 2, 6 pp. MR 19, 937.

Department of Mathematics, Brandeis University, Waltham, Massachusetts 02154

Department of Mathematics, Massachusetts Institute of Technology, Cambridge, MASSACHUSETTS 02139 